\title{
Stochastic Stokes' drift with inertia
}

\author{
By Kalvis M. Jansonst \\ Department of Mathematics, University College London, Gower Street, London \\ WC1E $6 B T, U K$
}

We consider both the effect of particle inertia on stochastic Stokes' drift, and also a related process which could be considered as a crude model of stochastic Stokes' drift driven by an eddy diffusivity. In the latter, the stochastic forcing is a stable Ornstein-Uhlenbeck process rather than Brownian motion. We show that the eddy Stokes' drift velocity has a peak at a non-zero value of the correlation time-scale for particles that have the same (limiting) diffusivity. For both of the models considered, this study shows that not only can stochastic Stokes' drift be used to sort particles with different diffusivities, but also it can be used to sort particles of the same diffusivities but with different particle masses or correlation time-scales. This effect may be important in particle sorting applications.

Keywords: Brownian motion, Stochastic Stokes' drift, particle sorting

\section{Introduction}

This study is a continuation of the work of Jansons \& Lythe (1998) on stochastic Stokes' drift, which is the modification of the classical Stokes' drift of a particle in a travelling wave due to the effect of Brownian motion (or some other random forcing with very short timescale correlations). In Jansons \& Lythe (1998), the particle inertia was neglected. In this study, we first consider, in $\S 2$, the effect of particle inertia on the stochastic Stokes' drift velocity. For simplicity, we still neglect the inertia in the fluid, so we can use the Stokes flow approximation to determine the drag on the particle, and so that this drag is not history dependent. This is a consistent approximation for a particle in a gas, where it is reasonable to assume that the particle density is much greater than that of the ambient fluid. For the motion of a particle in a liquid of similar density to that of the particle, the situation is much more complex, and the work here is not applicable, though may agree qualitatively in some cases.

Many authors have continued the investigation of various aspects of stochastic Stokes' drift. The first exact solution to Brownian motion forced Stokes' drift is due to Van Den Broeck (1999), who found an exact solution for an arbitrary wave in an integral form and simple exact solutions for a square wave and an impulse wave. Applications of stochastic Stokes' drift to ocean circulation have recently been considered by Restrepo (2006), and the techniques considered in this study appear to be relevant. Li Yu-Xiao et. al. (2001) studied the effect of asymmetric potentials on the stochastic Stokes' drift. Bena et. al. (2005) considered, both analytically and numerically, Stokes drift forced by a dichotomous Markov process.

$\dagger$ Email: stokesdrift@kalvis.com 
Also the theory of stochastic Stokes' drift applies to Brownian motors and tilting ratchets (see Reimann (2002)).

Jansons \& Lythe (1998) showed that particles of different diffusivities could be separated by the superposition of several waves with different directions, wavenumbers and frequencies. In such a system, it is possible to arrange for particles to drift in different directions depending on their diffusivities. We show here that it is also possible to separate particles of the same diffusivity but with different masses. It is not yet clear if this effect will have practical applications, but the early signs are promising.

In $\S 3$, we consider a related process of independent interest, and which could be considered a crude model of a particle moving in a travelling wave and small eddies, i.e. the random motion has short time-scale correlations. This is mathematically stochastic Stokes' drift forced not by Brownian motion but rather by a stable Ornstein-Uhlenbeck process. We show, for example, that it is possible for the stochastic Stokes' drift velocity to be increased by a careful choice of the correlation time-scale, even for particles with the same diffusivity in the long-time limit. Such an effect might be important in oceanographic applications of particle dispersion for example.

In $\S 4$, we compare the leading-order asymptotic approximations of the Stokes' drift for the two types of models considered here with Monte Carlo simulations, which agree well even for reasonably large values of the 'small' asymptotic parameter.

Finally, in $\S 5$, we consider applications of these results to particle separation schemes using multiple waves in dimensions greater than one.

\section{Stochastic Stokes' drift with inertia}

As shown by Jansons \& Lythe (1998), stochastic Stokes' drift in higher dimensions with many waves is, to leading order, a superposition of the contributions from each wave separately. This is because, in the leading-order calculation, cross-terms from different waves average to zero if they have either a different spatial or different temporal frequency. This is also true for the generalizations considered in this study. So, for simplicity, we consider the essentially one-dimensional system with a single wave, and comment on the higher-dimensional versions in $\S 5$.

The standard form of the Langevin equation for the velocity $U$ of an isotropic Brownian particle in a gas at rest is given by

$$
m \frac{d U}{d t}=-b^{-1} U+\eta,
$$

where $m$ and $b$ are respectively the particle mass and particle mobility, both assumed constant, and $\eta$ is a time-dependent noise term.

Note that assuming that $b$ is a constant follows from the assumption that we can neglect inertia effects in the motion of the fluid around the particle. This is a rational approximation in the case of a particle in a fluid, provided that the particle density is much greater than that of the fluid, which is normally so for a gas, but is rarely true for a liquid. In the case of a liquid, the particle drag term depends on the history of the particle's motion (see Hinch (1975)), and we need to include both fluid and particle inertia terms in the description of the motion, which we shall not 
do here. However, the results for a particle in a liquid are likely to be qualitatively similar to those of this study.

Turning (2.1) into an Itô stochastic differential equation we find

$$
d U_{t}=-\lambda U_{t} d t+\lambda \sigma d B_{t},
$$

where $B$ is a standard Brownian motion (i.e. a Wiener process), $\lambda=(b m)^{-1}$, $\sigma=(2 b K \mathcal{T})^{1 / 2}, K$ is Boltzmann's constant, and $\mathcal{T}$ is the (absolute) temperature. The long-time particle diffusivity is $\frac{1}{2} \sigma^{2}$.

We now introduce an additional velocity term $\varepsilon f$ due to some wave motion imposed on the fluid, giving

$$
d U_{t}=-\lambda\left(U_{t}-\varepsilon f\left(X_{t}, t\right)\right) d t+\lambda \sigma d B_{t},
$$

where $X_{t}$ is the particle position and $\varepsilon$ is a dimensionless constant, which will be used as a small parameter in the asymptotic analysis below. The stochastic Stokes' drift velocity is defined as

$$
V \equiv \lim _{t \rightarrow \infty} t^{-1}\left(X_{t}-X_{0}\right) .
$$

Using the Ergodic theorem we are able to replace this limit with an expectation of $U_{t}$, provided the starting phase of the wave is uniformly distributed.

For a wave with characteristic wavenumber $k$ and angular frequency $\omega$, the natural time-scales in this system are

$$
\lambda^{-1}, \quad \omega^{-1}, \quad \sigma^{-2} k^{-2},
$$

which are respectively the relaxation time-scale of the particle velocity, the period of the wave and the time for the particle to diffuse over a wavelength. These time-scales give two independent non-dimensional constants, in addition to $\varepsilon f_{0} k / \omega$, where $f_{0}$ is a typical value of $f$. We assume that all of the time-scales in (2.5) are comparable, and denote the common time-scale as $T$.

We can expand both $U$ and $X$ as a formal asymptotic series in $\varepsilon$, in the limit $\varepsilon \rightarrow 0$, namely

$$
U_{t}=U_{t}^{(0)}+\varepsilon U_{t}^{(1)}+\varepsilon^{2} U_{t}^{(2)}+\cdots
$$

and

$$
X_{t}=X_{t}^{(0)}+\varepsilon X_{t}^{(1)}+\varepsilon^{2} X_{t}^{(2)}+\cdots
$$

These local approximations are valid for an $o\left(\varepsilon^{-1} T\right)$ time range, which is sufficient for the determination of stochastic Stokes' drift as the exponential tails beyond this time range do not contribute to the leading-order asymptotic results. At times of order $\varepsilon^{-1} T$ some contributions switch order, but we do not need to address this point as we are not going to determine the first correction to the stochastic Stokes' drift in this study.

We now consider the formal $\varepsilon$ expansion an order at a time.

$$
\begin{gathered}
\text { (a) Order } \varepsilon^{0} \\
\lambda^{-1} d U_{t}^{(0)}=-U_{t}^{(0)} d t+\sigma d B_{t},
\end{gathered}
$$


which is a stable Ornstein-Uhlenbeck process and can be solved exactly in the form

$$
U_{t}^{(0)}=\mathrm{e}^{-\lambda t} W\left(\frac{1}{2} \lambda \sigma^{2} \mathrm{e}^{2 \lambda t}\right),
$$

where $W$ is a standard Brownian motion, with $W(0)=0$, which means that we have started $U^{(0)}$ with its stationary law. Note that $U_{t}^{(0)}$ has a zero mean.

Thus the autocorrelation of $U^{(0)}$ is given by

$$
\begin{gathered}
E\left[U_{s}^{(0)} U_{t}^{(0)}\right]=\frac{1}{2} \lambda \sigma^{2} \exp (-\lambda|s-t|) . \\
\text { (b) Order } \varepsilon^{1} \\
\lambda^{-1} \frac{d U_{t}^{(1)}}{d t}=-U_{t}^{(1)}+f\left(X_{t}^{(0)}, t\right) .
\end{gathered}
$$

Thus

$$
U_{t}^{(1)}=\lambda \int_{0}^{\infty} \exp (-\lambda \tau) f\left(X_{t-\tau}^{(0)}, t-\tau\right) d \tau
$$

(c) $\operatorname{Order} \varepsilon^{2}$

It is at this order we find the first contribution to the stochastic Stokes' drift.

$$
\lambda^{-1} \frac{d U_{t}^{(2)}}{d t}=-U_{t}^{(2)}+f^{\prime}\left(X_{t}^{(0)}, t\right) X_{t}^{(1)}
$$

where the dash denotes differentiation with respect to the first argument. Thus

$$
\frac{U_{t}^{(2)}}{\lambda}=\int_{0}^{\infty} \exp (-\lambda \tau) f^{\prime}\left(X_{t-\tau}^{(0)}, t-\tau\right) X_{t-\tau}^{(1)} d \tau
$$

This gives

$$
\frac{U_{t}^{(2)}}{\lambda}=\int_{0}^{\infty} \int_{\tau}^{\infty} \exp (-\lambda \tau) f^{\prime}\left(X_{t-\tau}^{(0)}, t-\tau\right) U_{t-\alpha}^{(1)} d \alpha d \tau
$$

and so

$$
\frac{U_{t}^{(2)}}{\lambda^{2}}=\int_{0}^{\infty} \int_{\tau}^{\infty} \int_{0}^{\infty} \exp (-\lambda(\tau+\beta)) f^{\prime}\left(X_{t-\tau}^{(0)}, t-\tau\right) f\left(X_{t-\alpha-\beta}^{(0)}, t-\alpha-\beta\right) d \beta d \alpha d \tau .
$$

This is about as far as it is useful to go in general, so we now focus on the particular case

$$
f(x, t)=u \cos (k x-\omega t+\phi),
$$

where $u, k, \omega$ and $\phi$ are constants. The phase $\phi$ of the wave will not alter the long-time limits of interest, so it is not important. However, for convenience, and to avoid starting transients, we choose $\phi$ to have a uniform distribution on $[0,2 \pi)$. Note that in this special case, $E\left[U_{t}^{(0)}\right]=E\left[U_{t}^{(1)}\right]=0$.

So the leading-order Stokes' drift is from $U^{(2)}$. From (2.16), we find

$$
\frac{U_{t}^{(2)}}{\lambda^{2} u^{2} k}=-\int_{0}^{\infty} \int_{\tau}^{\infty} \int_{0}^{\infty} \exp (-\lambda(\tau+\beta)) \sin \left(\phi_{s}\right) \cos \left(\phi_{c}\right) d \beta d \alpha d \tau,
$$


where $\phi_{s}=k X_{t-\tau}^{(0)}-\omega(t-\tau)+\phi$ and $\phi_{c}=k X_{t-\alpha-\beta}^{(0)}-\omega(t-\alpha-\beta)+\phi$. On averaging we find

$$
\frac{E\left[U_{t}^{(2)}\right]}{\frac{1}{2} \lambda^{2} u^{2} k}=-\int_{0}^{\infty} \int_{\tau}^{\infty} \int_{0}^{\infty} \exp (-\lambda(\tau+\beta)) E\left[\sin \left(\phi_{s}-\phi_{c}\right)\right] d \beta d \alpha d \tau .
$$

This is exact, rather than a long-time limit, since we chose $\phi$ to have a uniform distribution, so the time $t=0$ is not special.

Note that

$$
\phi_{s}-\phi_{c}=k\left(X_{t-\tau}^{(0)}-X_{t-\alpha-\beta}^{(0)}\right)+\omega(\tau-\alpha-\beta),
$$

which is Gaussian, and so $E\left[\sin \left(\phi_{s}-\phi_{c}\right)\right]$ can be evaluated exactly. From (2.9), we see that

$$
C(t-s) \equiv E\left[\left(X_{t}^{0}-X_{s}^{0}\right)^{2}\right]=\sigma^{2}\left(|t-s|+\frac{\left(\mathrm{e}^{-\lambda|t-s|}-1\right)}{\lambda}\right) .
$$

Thus

$$
E\left[\sin \left(\phi_{s}-\phi_{c}\right)\right]=\exp \left(-\frac{1}{2} k^{2} C(\tau-\alpha-\beta)\right) \sin (\omega(\tau-\alpha-\beta)) .
$$

Putting everything together, we find from (2.19) the stochastic Stokes drift velocity, $V$, to leading-order in $\varepsilon$ :

$\frac{V}{\frac{1}{2} \varepsilon^{2} \lambda^{2} u^{2} k}=\int_{0}^{\infty} \int_{\tau}^{\infty} \int_{0}^{\infty} \exp \left(-\lambda(\tau+\beta)-\frac{1}{2} k^{2} C(\alpha+\beta-\tau)\right) \sin (\omega(\alpha+\beta-\tau)) d \beta d \alpha d \tau$.

We may simplify this a little, to give

$$
V=\frac{1}{2} \varepsilon^{2} \lambda u^{2} k \int_{0}^{\infty} \int_{0}^{\infty} \exp \left(-\lambda \beta-\frac{1}{2} k^{2} C(\alpha+\beta)\right) \sin (\omega(\alpha+\beta)) d \beta d \alpha .
$$

We should expect (2.24) to reduce to the result of Jansons \& Lythe (1998) in the limit $\lambda \rightarrow \infty$, i.e. in the zero mass limit. Taking this limit reduces (2.24) to

$$
V=\frac{1}{2} \varepsilon^{2} u^{2} k \int_{0}^{\infty} \exp \left(-\frac{1}{2} k^{2} C(\alpha)\right) \sin (\omega \alpha) d \alpha,
$$

and

$$
C(\alpha)=\sigma^{2}|\alpha| .
$$

Equations (2.25) and (2.26) agree exactly with the penultimate line in the calculation of Jansons \& Lythe (1998), but in a slightly different notation.

To leading order

$$
\lim _{t \rightarrow \infty} \frac{\operatorname{Var}\left[X_{t}\right]}{t}=\sigma^{2} .
$$

We could easily determine the first correction to the variance of position, but we do not present this result here as it was felt that it was a little too messy to be of interest. 


\section{Eddy Stokes'drift}

In this section, we consider a closely-related process to the stochastic Stokes' drift with inertia process of $\S 2$. This process is stochastic Stokes' drift forced by a stable Ornstein-Uhlenbeck process rather than Brownian motion. The governing equation (using notation as close to $\S 2$ as possible) is

$$
\frac{d X_{t}}{d t}=U_{t}+\varepsilon f\left(X_{t}, t\right)
$$

where $U_{t}$ satisfies (2.2), but, of course, does not have the same physical interpretation. This process could be considered as a crude (or qualitative) description of stochastic Stokes' drift with the randomness coming from eddy diffusivity.

In the case $f(x, t)=u \cos (k x-\omega t+\phi)$, the stochastic Stokes' drift, to leading order, is given by

$$
V=\frac{1}{2} \varepsilon^{2} u^{2} k \int_{0}^{\infty} \sin (\omega t) \exp \left(-\frac{1}{2} k^{2} C(t)\right) d t
$$

which follows from an argument that is a simpler version of that in $\S 2$, and is the same as that of Jansons \& Lythe (1998) but with $\sigma^{2} t$ replaced by $C(t)$, so will not be given here. In this case, it is also trival to see that the $\lambda \rightarrow \infty$ limit of this result is exactly the same as that for Brownian forcing in Jansons \& Lythe (1998).

We now determine the leading-order correction to the long-time limit of the variance of position. This will appear at order $\varepsilon^{2}$. We take $X_{0}^{(n)}=0$ for all $n$. Note that, to the required order, $t^{-1} E\left[X_{t}\right] \rightarrow 0$, as $t \rightarrow \infty$, so we need only to determine

$E\left[X_{t}^{2}\right]=E\left[\left(X_{t}^{(0)}\right)^{2}\right]+2 \varepsilon E\left[X_{t}^{(0)} X_{t}^{(1)}\right]+\varepsilon^{2}\left(E\left[\left(X_{t}^{(1)}\right)^{2}\right]+2 E\left[X_{t}^{(0)} X_{t}^{(2)}\right]\right)+\cdots$,

which we consider term by term.

$$
t^{-1} E\left[\left(X_{t}^{(0)}\right)^{2}\right]=t^{-1} C(t) \rightarrow \sigma^{2},
$$

as $t \rightarrow \infty$;

$$
t^{-1} E\left[X_{t}^{(0)} X_{t}^{(1)}\right] \rightarrow 0
$$

as $t \rightarrow \infty$;

$$
\begin{aligned}
t^{-1} E\left[\left(X_{t}^{(1)}\right)^{2}\right] & =t^{-1} u^{2} \int_{0}^{t} \int_{0}^{t} E\left[\cos \left(k X_{\alpha}^{(0)}-\omega \alpha\right) \cos \left(k X_{\beta}^{(0)}-\omega \beta\right)\right] d \alpha d \beta \\
& \rightarrow u^{2} \int_{0}^{\infty} \cos (\omega t) \exp \left(-\frac{1}{2} k^{2} C(t)\right) d t
\end{aligned}
$$

as $t \rightarrow \infty$;

$$
t^{-1} E\left[X_{t}^{(0)} X_{t}^{(2)}\right] \rightarrow 0
$$

as $t \rightarrow \infty$

Thus

$$
\lim _{t \rightarrow \infty} \frac{\operatorname{Var}\left[X_{t}\right]}{t}=\sigma^{2}+\varepsilon^{2} u^{2} \int_{0}^{\infty} \cos (\omega t) \exp \left(-\frac{1}{2} k^{2} C(t)\right) d t+\cdots
$$




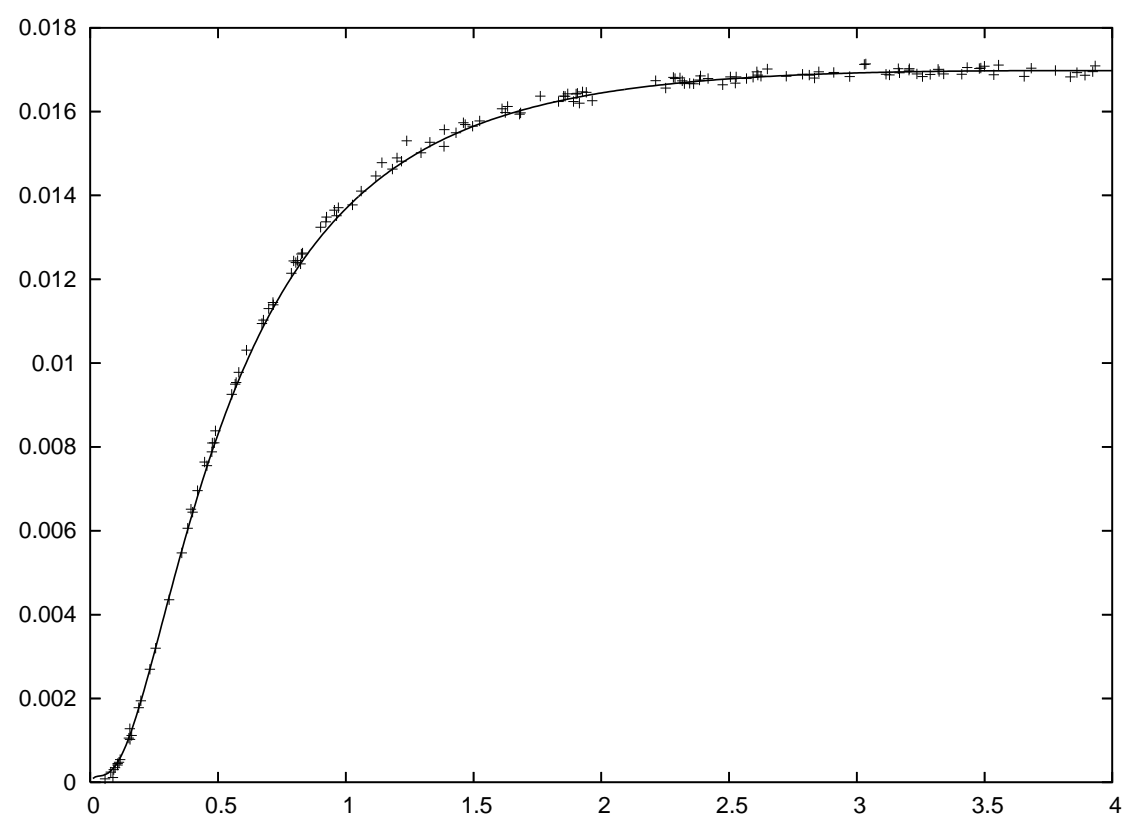

Figure 1. Stochastic Stokes' drift velocity against $\lambda$ for $\varepsilon=0.2$, and all other parameters set equal to 1 . The curve is the leading-order asymptotic result and the points are Monte Carlo results.

We can also consider the limit $\lambda \rightarrow \infty$ of this result, giving the corresponding result for Brownian forcing, though this was not determined by Jansons \& Lythe (1998) in their study of this process. In this limit, we find

$$
\lim _{t \rightarrow \infty} \frac{\operatorname{Var}\left[X_{t}\right]}{t}=\sigma^{2}+\varepsilon^{2} \frac{2 u^{2} k^{2} \sigma^{2}}{k^{4} \sigma^{4}+4 \omega^{2}}+\cdots .
$$

\section{Numerical results}

To determine how small $\varepsilon$ needs to be for the asymptotic results to be a good approximation to the exact results, we perform some Monte Carlo simulations. In the simulations shown here, we use the stochastic Euler method with $d t=0.001$ and all parameters other than $\varepsilon$ set equal to 1 . These simulations are run to $t=10^{8}$ to control the variance sufficiently for comparison of the time-averaged stochastic Stokes' drift.

We present numerical confirmation of the results of both $\S 2$ and $\S 3$.

In the case of stochastic Stokes' drift with inertia, and for $\varepsilon=0.2$, the agreement is excellent and is shown in Figure 1, though the (predicted) variance leads to a little scatter around the asymptotic result. This could have been reduced by running the Monte Carlo simulations up to a a larger time $t$, but as it was each point took 5 hours on a $3 \mathrm{GHz}$ Intel $\mathrm{CPU}$.

When $\varepsilon=0.5$ for this model, the scatter of the Monte Carlo results is reduced (see Figure 2) and a small departure from the asymptotic approximation can be seen, but it is still small enough for the asymptotic result to be useful in applications. 


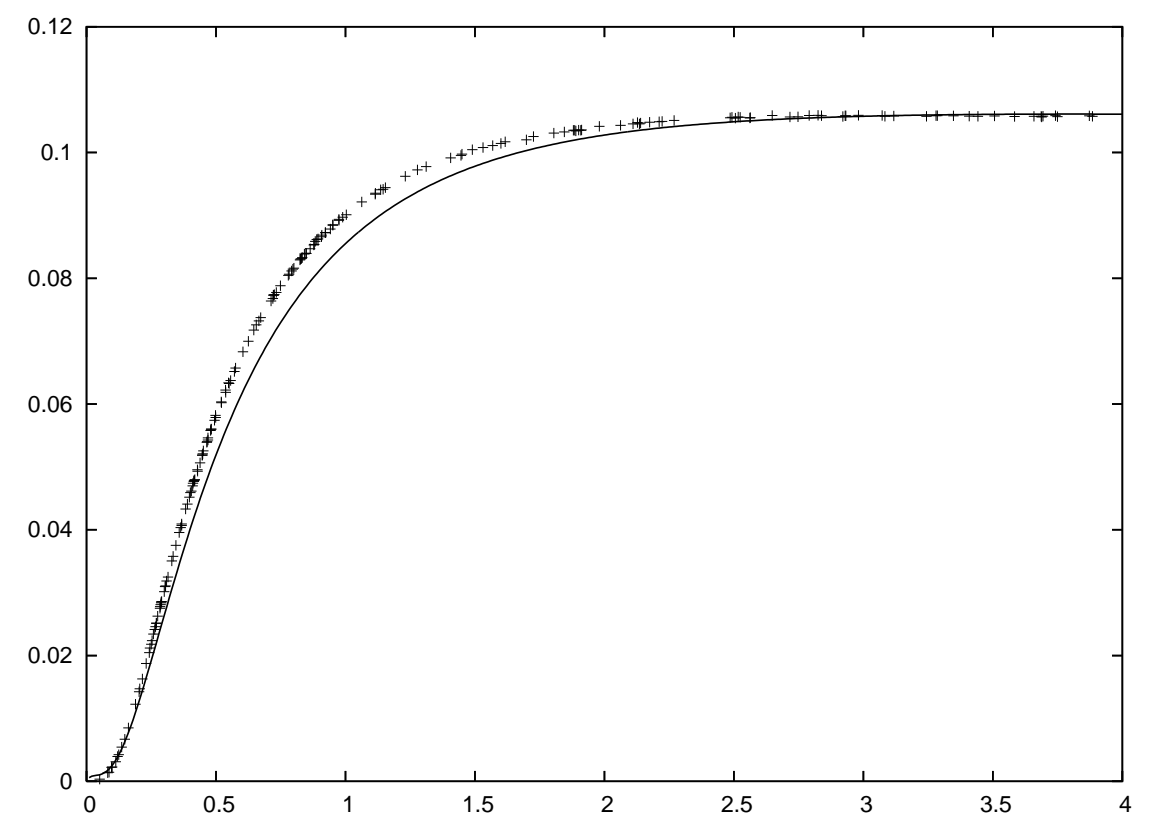

Figure 2. Stochastic Stokes' drift velocity against $\lambda$ for $\varepsilon=0.5$, and all other parameters set equal to 1 . The curve is the leading-order asymptotic result and the points are Monte Carlo results.

The agreement between Monte Carlo results and asymptotic results is much the same for the case of eddy Stokes' drift (§3). However, for this model there is a peak in the Stokes' drift, which was not seen in the model of $\S 2$, and is shown in Figures 3 and 4 for $\varepsilon=0.2$ and $\varepsilon=0.5$ respectively. Thus the eddy Stokes' drift at finite $\lambda$ can be larger than that of the $\lambda \rightarrow \infty$ limit (i.e. larger than for the Brownian Stokes' drift result). This does not appear to occur in the model of $\S 2$.

Finally, we plot the first correction to the large-time limit of the particle variance for eddy Stokes' drift in Figure 5.

\section{Multiple waves and higher dimensional results}

In the work of Jansons \& Lythe (1998), they considered stochastic Stokes' drift in several dimensions. In this case, it was possible to sort particles by a combination of waves in different directions and different spatial or temporal frequencies. At leading order in the Stokes' drift, such combinations of waves act independently on the particle as the cross-terms average to zero in the long-time limit. Thus the modifications to the classical Stokes' drift due to diffusion is different for each of the wave components and so the linear combinations of these drifts will in general result in particles of different diffusivities diffusing in different directions. This leads to an interesting continuous particle sorting method.

In the current study too, the higher dimensional versions work in exactly the same way, as for the same reason all cross-terms due to different waves average to zero, at leading order, provided that they have either different spatial or temporal 


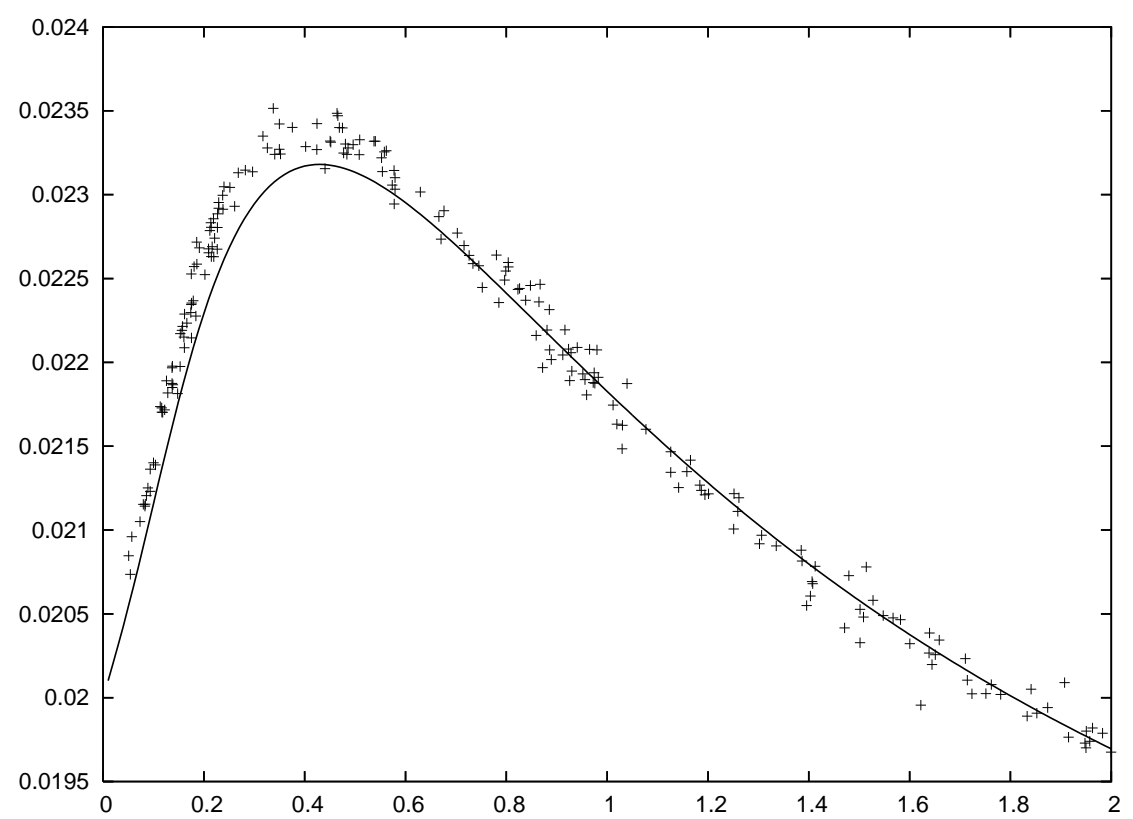

Figure 3. Eddy Stokes' drift velocity against $\lambda$ for $\varepsilon=0.2$, and all other parameters set equal to 1 . The curve is the leading-order asymptotic result and the points are Monte Carlo results.

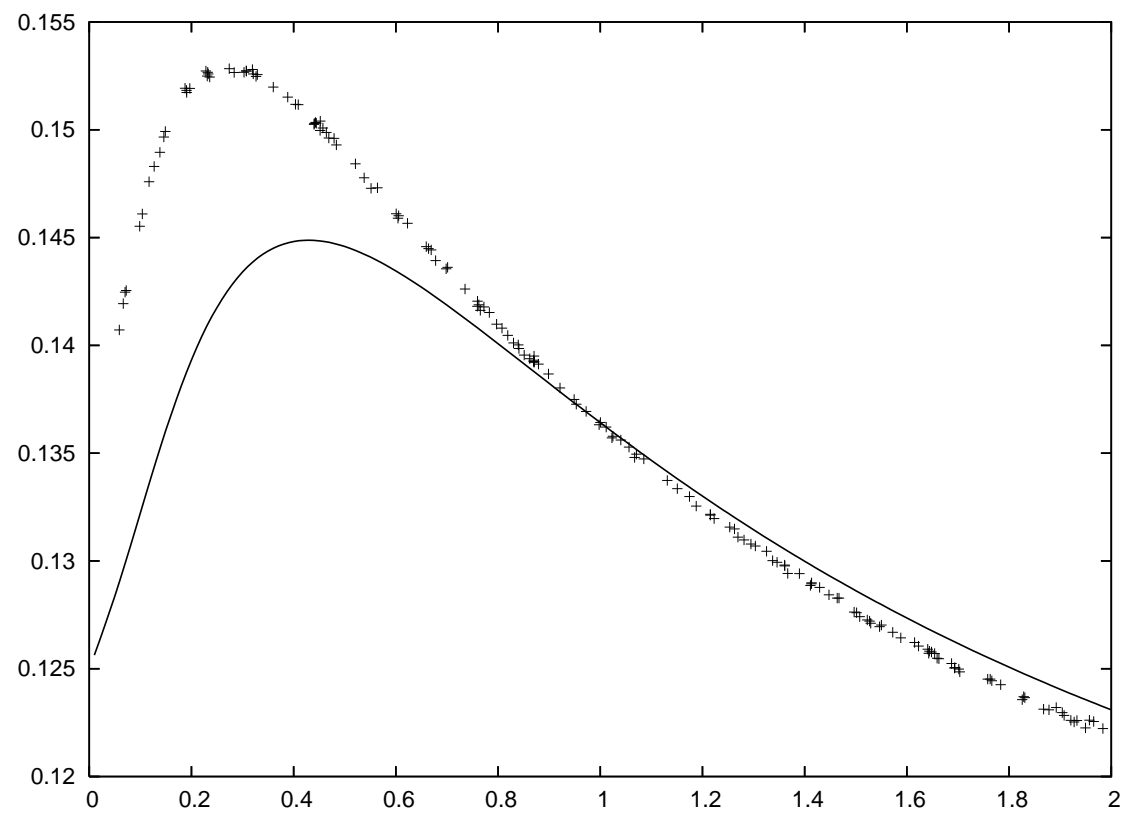

Figure 4. Eddy Stokes' drift velocity against $\lambda$ for $\varepsilon=0.5$, and all other parameters set equal to 1 . The curve is the leading-order asymptotic result and the points are Monte Carlo results. 


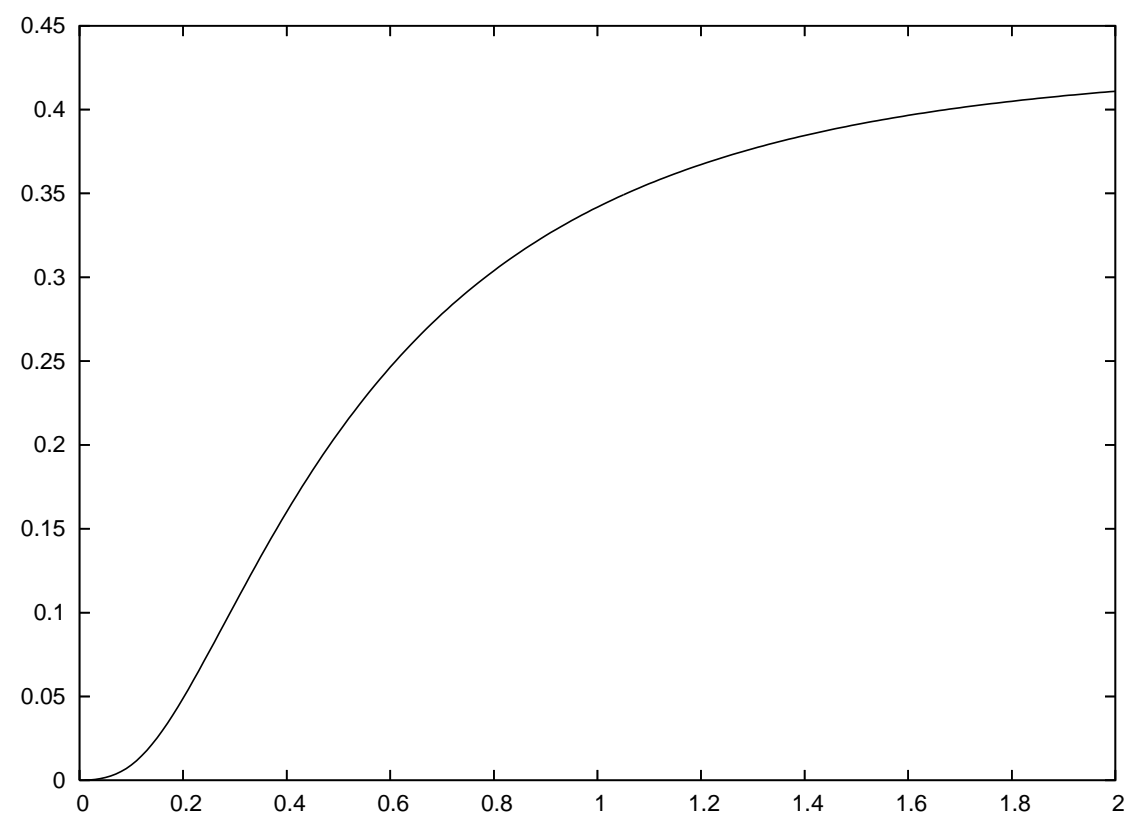

Figure 5. The first correction to the variance coefficient for the particle position for eddy Stokes' drift against $\lambda$.

frequencies. Thus again the resulting leading-order stochastic Stokes' drift is just a linear combination of the stochastic Stokes' drifts of each component.

This implies that, in the case of stochastic Stokes' drift with inertia (see §2), it is possible to sort particles that have the same diffusivity but different masses, by arranging them to have different directions for the resulting Stokes' drift. Whether this has practical applications remains to be seen, but it certainly looks hopeful.

Equally, in the case of eddy Stokes' drift (see §3), particles with the same diffusivity in the long-time limit but with different correlation times can be sorted by arranging the resulting Stokes' drift to be in different directions.

\section{Conclusions}

In this study, we have extended the work of Jansons \& Lythe (1998) in two directions. Firstly in $\S 2$, we consider stochastic Stokes' drift including the effect of particle inertia, and secondly, we consider stochastic Stokes' drift forced by a stable Ornstein-Uhlenbeck process rather than Brownian motion. Both of these results are to leading-order in the strength $\varepsilon$ of the wave motion, and the stochastic Stokes' drift appears at order $\varepsilon^{2}$. These results extend trivially to higher dimensions and many waves, as the cross-terms for waves of different spatial or temporal frequencies average to zero. Thus the 'fanout' of particles of different diffusivities observed by Jansons \& Lythe (1998) extends to particles of the same (long-time limit) of diffusivity but with different masses in the case of the model in $\S 2$ and different correlation times in the case of the model of $\S 3$. In the case of eddy Stokes' drift (see $\S 3)$, we also compute the long-time limit of the variance of particle positions. The 
short correlation time limit of this result applies directly to the Brownian motion forcing case studied by Jansons \& Lythe (1998).

The agreement of these results with Monte Carlo simulations is impressive (see $\S 4$ ), and shows that, for practical applications, the leading-order asymptotic approximations are sufficient, even when the 'small' asymptotic parameter $\varepsilon=0.5$. Note that this is partly due to the asymptotic expansion being naturally in $\varepsilon^{2}$ rather than $\varepsilon$, as replacing $\varepsilon$ with $-\varepsilon$ is equivalent to a phase shift, and does not effect the stochastic Stokes' drift velocity.

It appears that Van Den Broeck's (1999) exact method could be extended to consider the system of this study, but it is less clear if there are simple exact expressions even for a square wave.

One extension of these results, which would be of interest, is to consider the behaviour of flexible particles that are large enough to feel different parts of the wave. The simplest such example would be a flexible dumbbell, consisting of two point particles connected by a linear spring.

\section{References}

Jansons, K.M. and Lythe, G.D. 1998 Stochastic Stokes' Drift, Phys Rev. Let. 81, 15, 3136-3139.

Van Den Broeck, C. 1999 Stokes' drift: An exact result, Europhsyics Let. 46 (1), 1-5.

Restrepo, J.M. 2006 Wave breaking dissipation in the wave-driven ocean circulation, preprint.

Li Yu-Xiao, Cao Tian-Guang, Wu Xi-Zhen and Zhuo Yi-Zhong 2001 Effects of asymmetry of the potential on stochastic Stokes' drift, Chinese Phys. Lett. 18, 488-490.

Bena, I., Kawai, R., Van den Broeck, C. and Lindenberg, K. 2005 Stokes' drift and hypersensitive response with dichotomous markov noise, To appear in Fluctuation and Noise Letter.

Reimann, P. 2002 Brownian motors: noisy transport far from equilibrium, Physics Reports 361, 57-265.

Hinch, E.J. 1975 Applications of the Langevin equation to fluid suspensions, J. Fluid Mech. 72, 499-511.

\$Id: StokesDrift.tex 2343 2006-09-06 12:44:57Z kalvis \$ 